\title{
The analogy between the timing of renal replacement therapy and mechanical circulatory intervention
}

\author{
Rohit K. Singal, MD, MSc, FRCSC, and Rakesh C. Arora, MD, PhD, FRCSC
}

\footnotetext{
From the Department of Surgery, University of Manitoba, and Cardiac Sciences Program, St Boniface Hospital, Winnipeg, Manitoba, Canada.

Disclosures: R.C.A. has an unrestricted educational grant from Pfizer Canada Inc, and has received honoraria from Mallinckrodt Pharmaceuticals for work unrelated to this article. The other author has nothing to disclose with regard to commercial support.

Received for publication May 5, 2017; accepted for publication May 8, 2017; available ahead of print June 17, 2017.

Address for reprints: Rohit K. Singal, MD, MSc, FRCSC, St Boniface Hospital, CR3030 - 369 Tache Ave, Winnipeg, Manitoba R2H 2A6, Canada (E-mail: rsingal@sbgh.mb.ca).

J Thorac Cardiovasc Surg 2017;154:865-6

$0022-5223 / \$ 36.00$

Copyright (C) 2017 by The American Association for Thoracic Surgery

http://dx.doi.org/10.1016/j.jtcvs.2017.05.013
}

Understanding outcomes after mechanical circulatory support (MCS) is important for our field. Abadeer and colleagues have provided insight on the impact of acute kidney injury (AKI) in the setting of short-term mechanical circulatory support (ST-MCS). ${ }^{1}$ They applied standard definitions of renal injury and recovery ${ }^{2}$ to their vast experience in ST-MCS at their center. Limitations of a retrospective, single-center design with the inability to access certain relevant data (ie, creatinine before shock state) notwithstanding, they confirmed that greater than half of patients supported with ST-MCS are likely to experience some degree of AKI and that those requiring dialysis have a pronounced risk of increased 1-year mortality. The "glass half full" interpretation is that patients with AKI not requiring dialysis had high rates of renal recovery $(>82 \%)$ and followed a survival trajectory similar to those without AKI. This cautions us against overly negative prognostication in these cases, especially given the implications on consideration of long-term MCS survivorship. It is largely a hypothesis-generating notion that requires prospective validation.

As in many centers, the timing of renal replacement therapy (RRT) once AKI was suspected was largely nonstandardized with decisions made case by case with their nephrology partners. To date, the timing of RRT has been hotly contested with some advocating for therapy early in the presence of AKI as opposed to waiting for "traditional indications" to develop. ${ }^{3}$ Proponents of early therapy suggest that intervening before the negative consequences of established renal failure (eg, metabolic derangement, venous congestion) on other organ systems may result in better outcomes. Specifically, what effect does established renal failure have on the heart and other organs? The question remains unanswered, although much effort to answer it is being undertaken.

Paradoxically, a similar conundrum exists in the reverse as suggested by the investigators in this analysis. What effect does established cardiac failure have on the kidney?

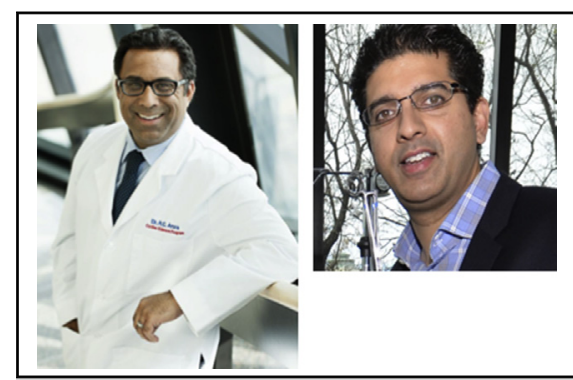

Drs Rakesh C. Arora, MD, PhD, FRCSC, and Rohit K Singal, MD, MSc, FRCSC

Central Message

It is time for prospective, collaborative studies to address the optimization of outcomes in patients who receive MCS.

See Article page 856 .

Although the pathophysiologic answer is perhaps more intuitive, the timing of cardiac intervention is not. The salient point lies in Abadeer and colleagues' examination of the predictors of renal failure. Of 2 independent survival outcome predictors, serum creatinine before the initiation of ST-MCS was associated with a greater risk for every $1 \mathrm{mg} / \mathrm{dL}$ increase. Would earlier use of ST-MCS have detected patients before the increase in serum creatinine and prevented subsequent renal failure? Although logical, this implication is not sufficient in isolation to promote early resuscitation with ST-MCS. As with RRT, the question regarding timing of ST-MCS remains.

It is increasingly clear that we need better information to systematically address these issues. Our critical care and renal colleagues are doing this with trials such as the Standard versus Accelerated Renal Replacement Therapy in Critically Ill Patients with Acute Kidney Injury (NCT02568722 and pilot study ${ }^{4}$ ). Leaders, such as the group from this report, have repeatedly demonstrated the ability, success, risk, and failure of ST-MCS. However, this study highlights that the MCS community is reaching the limit of what can be learned from retrospective, single-center experiences. We must now move to the next level of inquiry prospectively and with specific hypothesis testing via inter-institutional collaboration to specifically define this role of this therapy. Would ST-MCS at an earlier 
stage of hemodynamic derangement lead to better outcomes? It is definitely plausible, but to know with certainty we have to work together to collect the data.

\section{References}

1. Abadeer AI, Kurlansky P, Chiuzan C, Truby L, Radhakrishan J, Garan R, et al. Importance of stratifying acute kidney injury in cardiogenic shock resuscitated with mechanical ciruculatory support therapy. J Thorac Cardiovasc Surg. 2017;154: 856-64.e4.
2. Kidney Disease: Improving Global Outcomes (KDIGO) CKD Work Group. KDIGO clinical practice guideline for the evaluation and management of chronic kidney disease. Kidney Int Suppl. 2012;2:1-138.

3. Karvellas CJ, Farhat MR, Sajjad I, Mogensen SS, Leung AA, Wald R, et al. A comparison of early versus late initiation of renal replacement therapy in critically ill patients with acute kidney injury: a systematic review and meta-analysis. Crit Care. 2011;15:R72.

4. Wald R, Adhikari NKJ, Smith OM, Weir MA, Popess K, Cohen A, et al. Comparison of standard and accelerated initiation of renal replacement therapy in acute kidney injury. Kidney Int. 2015;88:897-904. 